\title{
ON THE COMPOSITION FUNCTIONS OF NILPOTENT LIE GROUPS
}

\author{
KUO-TSAI CHEN
}

This note contains a proof of the theorem:

If the composition functions of a real or complex Lie group are polynomials, then it is nilpotent.

The converse theorem is found among E. Cartan's works [2] and can be also shown through the Baker-Hausdorff formula in a rather straightforward fashion (see [1]).

Let $(5)$ be a real or complex Lie group with Lie algebra g. Choose for $\mathrm{g}$ a base $X_{1}, \cdots, X_{n}$, dual to which there is a base $\omega_{1}, \cdots, \omega_{n}$ of the Maurer-Cartan forms. Denote by $\mathrm{x}=\left(x_{1}, \cdots, x_{n}\right)$ the corresponding canonical coordinate system and by $f_{i}(\mathbf{x}, \mathrm{y}), i=1, \cdots, n$, the composition functions with respect to the coordinate system. If $f_{i}(\mathbf{x}, \boldsymbol{y}), i=1, \cdots, n$, are polynomials, we are going to prove that (5) is nilpotent.

For $X \in \mathfrak{g}$, define $R(X)$ to be the linear transformation (operating on the right side) of the vector space of $\mathfrak{g}$ such that, for $Y \in \mathfrak{g}$,

$$
Y R(X)=[Y, X]=Y X-X Y .
$$

Allow the vector space of $\mathrm{g}$ to be normed so that the norm $|R(X)|$ of $R(X)$ is defined.

It is known that, about the identity $e$ of $B$, the Maurer-Cartan forms

$$
\omega_{i}(\mathbf{x}, d \mathbf{x})=\sum A_{i j}(\mathbf{x}) d x_{j}, \quad i=1, \cdots, n,
$$

are explicitly given through the formulas

$$
\sum A_{j k}(\mathrm{x}) X_{j}=X_{k} \frac{\exp R(X)-1}{R(X)}, \quad k=1, \cdots, n,
$$

where $X=\sum x_{i} X_{i}$.

We assert that, about $e$, the left invariant infinitesimal transformations $X_{i}=\sum H_{i j}(\mathbf{x}) \partial / \partial x_{j}, i=1, \cdots, n$, can be explicitly given through the formulas

$$
\sum H_{i j}(\mathrm{x}) X_{j}=X_{i} \frac{R(X)}{\exp R(X)-1} .
$$

Presented to the Society, November 27, 1953 under the title On a Cartan's theorem and its converse; received by the editors January 26, 1957. 
In fact, if $\bar{X}_{i}=\sum H_{i j}(\mathbf{x}) \partial / \partial x_{j}, i=1, \cdots, n$, where the $H_{i j}$ are defined by the preceding equation, then we have about $e$,

$$
\begin{aligned}
\sum \omega_{i}\left(\bar{X}_{j}\right) X_{i} & =\sum A_{i, k}(\mathbf{x}) H_{j k}(\mathbf{x}) X_{i} \\
& =\sum H_{j k}(\mathbf{x}) X_{k} \frac{\exp R(X)-1}{R(X)} \\
& =X_{j} \frac{R(X)}{\exp R(X)-1} \frac{\exp R(X)-1}{R(X)} .
\end{aligned}
$$

Since the series respectively represented by $R(X) /(\exp R(X)-1)$ and $(\exp R(X)-1) / R(X)$ converge both absolutely, we obtain $\sum \omega_{i}\left(\bar{X}_{j}\right) X_{i}=X_{j}$, i.e. $\omega_{i}\left(\bar{X}_{j}\right)=\delta_{i j}$. Therefore each $\bar{X}_{i}$ coincides with $X_{i}$ about $e$.

On the other hand, we observe that each $H_{i j}(x)$ is equal to $\left(\partial f_{i}(x, y) / \partial y_{j}\right)_{y=0}$ which is a polynomial, say, of degree $\leqq N$. Let $R(X) /(\exp R(X)-1)$ be expanded in the series $\sum_{p=0}^{\infty} B_{p}[R(X)]^{p}$ and set $B_{p} X_{i}[R(X)]^{p}=\sum H_{i j p}(\mathbf{x}) X_{j}$. Then each $H_{i j p}(\mathbf{x})$ is a homogeneous polynomial of degree $p$, and

$$
H_{i j}(\mathbf{x})=\delta_{i j}+\sum_{p=1}^{\infty} H_{i j p}(\mathbf{x}) .
$$

We conclude that $B_{p} X_{i}[R(X)]^{p}=0, i=1, \cdots, n$, and therefore $B_{p} Y[R(X)]^{p}=0$ for any $Y \in g$ when $p>N$. Since there exists $q>N$ with $B_{q} \neq 0$, this yields $Y[R(X)]^{q}=0$ for any $X, Y \in \mathfrak{g}$. According to a theorem due to Engel (see [3]), $\mathfrak{g}$ is nilpotent. Hence $B$ is nilpotent.

\section{BibLIOGRAPHY}

1. G. Birkhoff, Analytical groups, Trans. Amer. Math. Soc. vol. 43 (1938) pp. 61101.

2. E. Cartan, Les représentations linéaires des groupes de Lie, J. Math. Pures Appl. vol. 17 (1938) pp. 1-12.

3. H. Zassenhaus, Über Liesche Ringe mit Primzahlcharakteristik, Abh. Math. Sem. Hanischen Univ. vol. 13 (1939) pp. 1-100.

UNIVERSITY OF HoNG KONG 